\title{
Deux clavicules de marmotte épigravettiennes incisées provenant des grottes Verdi de Pradis (Alpes italiennes)
}

Two Epigravettian engraved marmot clavicles from the Grottes Verdi de Pradis (Alpes italiennes)

Fabio Gurioli, Giorgio Bartolomei, Nicola Nannini, Marco Peresani et Matteo Romandini

\section{OpenEdition}

\section{Journals}

Édition électronique

URL : http://journals.openedition.org/paleo/2180

ISSN : 2101-0420

Éditeur

SAMRA

\section{Édition imprimée}

Date de publication : 1 décembre 2011

Pagination : $311-318$

ISSN : $1145-3370$

\section{Référence électronique}

Fabio Gurioli, Giorgio Bartolomei, Nicola Nannini, Marco Peresani et Matteo Romandini, « Deux clavicules de marmotte épigravettiennes incisées provenant des grottes Verdi de Pradis (Alpes italiennes) », PALEO [En ligne], 22 | 2011, mis en ligne le 19 avril 2012, consulté le 20 avril 2019. URL: http://journals.openedition.org/paleo/2180

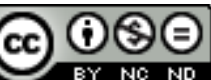

PALEO est mis à disposition selon les termes de la licence Creative Commons Attribution - Pas d'Utilisation Commerciale - Pas de Modification 4.0 International. 


\title{
DEUX CLAVICULES
}

\section{DE MARMOTTE EPIGRAVETTIENNES INCISÉES PROVENANT DES GROTTES VERDI DE PRADIS (Alpes italiennes)}

\author{
Fabio GURIOLI(1), Giorgio BARTOLOMEI ${ }^{(2)}$, Nicola NANNINI ${ }^{(1)}$, \\ Marco PERESANI ${ }^{(1)}$ et Matteo ROMANDINI ${ }^{(1)}$
}

\begin{abstract}
Résumé : Cet article présente l'étude de deux clavicules de marmotte mises au jour en 1970-1971, dans les niveaux tardiglaciaires des Grottes Verdi de Pradis, qui livrent une industrie attribuable à l'Epigravettien récent. Ces deux clavicules droites ont été incisées, l'une de quatre encoches, l'autre de cinq, parmi lesquelles quatre montrent une reprise de l'encoche suivant un axe différent. Après avoir discuté l'origine de ces pièces, nous montrons leur caractère unique pour le Paléolithique supérieur des Alpes et nous proposons de les interpréter comme des objets de caractère non utilitaire.
\end{abstract}

Mots-clés : Marmota marmota, clavicules, gravures, Epigravettien, Alpes italiennes.

Abstract: Two Epigravettian engraved marmot clavicles from the Grottes Verdi de Pradis (Alpes italiennes). This note presents the results of the surface analyses carried out on two marmot clavicles discovered during the 1970-71 archaeological campaigns at the Late-Glacial Epigravettian site Grotte Verdi di Pradis. Both the clavicles are right and are engraved with four scores the first one and with five scores the second one, four of which were successively crossed with twisted direction. After a discussion about their origin, we point out the uniqueness of these pieces in the Late Palaeolithic of the Alps, interpretable like non-utilitarian objects.

Key-words: Marmota marmota, clavicles, engravings, Epigravettian, Italian Alps.

\section{Introduction}

Bien qu'occasionnelle, l'exploitation de la Marmotte, à but alimentaire ou économique, a été documentée en Europe, notamment sur des sites des Alpes et des Apennins. Des témoignages sont présents dès le Paléolithique moyen et jusqu'à la fin du Tardiglaciaire, voire au-delà (Patou-Mathis 1987). Si, pour les périodes les plus reculées, les accumulations anthropiques des restes squelettiques de ce rongeur sont extrêmement sporadiques, durant le Tardiglaciaire, l'importance économique de l'exploitation de la Marmotte est plus marquée dans certaines régions. C'est notamment le cas des Alpes occidentales françaises et du Jura, dont les hauts plateaux, caractérisés par des conditions morphologiques et écologiques spécifiques, ont permis, pendant le Magdalénien et l'Azilien, la mise en place de systèmes logistiques selon un rythme saisonnier (Tillet 2001 ; Tomé et Chaix 2003 ; Monin et al. 2006 ; Griggo et al. com. orale). Les études archéozoologiques menées sur les restes de marmottes, témoignent essentiellement des modalités de la boucherie, du dépouillement à la consommation, en passant par le dépeçage (Patou-Mathis 1987 ; Patou-Mathis et al. 1991 ; Tomé et Chaix 2003).

En revanche, peu de matériel fournit des évidences permettant de conférer une dimension symbolique aux modifications anthropiques souvent découvertes sur des portions squelettiques bien précises. Les plus anciennes traces de transformation à fonction non utilitaire ont été

(1) Università di Ferrara, Dipartimento di Biologia ed Evoluzione, Sezione di Paleobiologia, Antropologia e Preistoria, Corso Ercole I d'Este 32, I-44100 Ferrara, Italie - fabio.gurioli@unife.it, nicolanannini@libero.it, marco.peresani@unife.it, matteo.romandini@unife.it (2) Via Trissino 15, 36100 Vicenza, Italie - studiobartolomei@tiscali.it 
observées sur une incisive sciée, présente dans les niveaux châtelperroniens de la grotte du Renne à Arcy-surCure (Yonne, France) (d'Errico et al. 1998). Pour le Magdalénien, Bollinger et Müller (2005) décrivent une vingtaine d'incisives avec des traces de sciage, sur certains sites magdaléniens de l'arc jurassien des Alpes du Nord : en Allemagne à Petersfels (Poplin 1983) et en Suisse à Monruz (Leesch et al. 2004) et Champréveyres (Leesch et al. 2004), à Kesslerloch et dans la grotte de Romains (Desbrosse 1972). II faut également compter avec les incisives perforées provenant de Monruz ainsi que de l'abri de Campalou en France (Brochier et Brochier 1973).

Dans les grottes Verdi de Pradis, sur le haut plateau homonyme, dans les Préalpes Carniques (Alpes orientales italiennes), deux clavicules de Marmotte, présentant des entailles incisées qui pourraient témoigner d'une fonction non utilitaire, sont décrites dans cette note. Elles ont été découvertes à $520 \mathrm{~m}$ d'altitude (fig. 1).

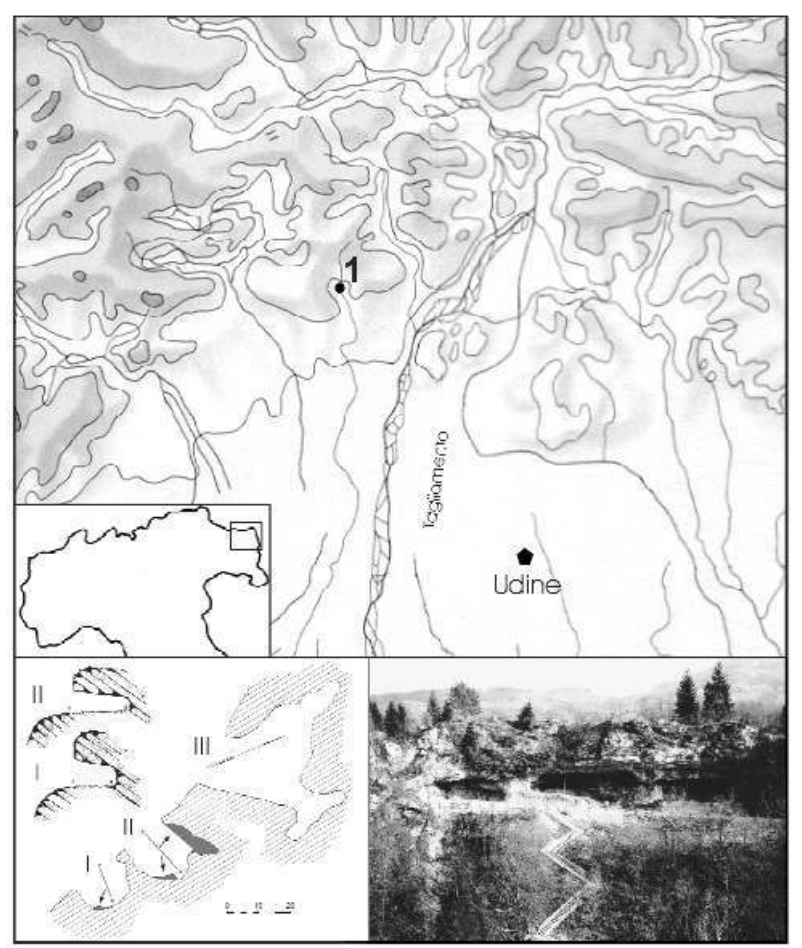

Figure 1 - Localisation des grottes Verdi de Pradis dans les Préalpes Carniques. En bas à gauche, plan des trois abris, avec section des abris I et II. A droite, les trois abris vus depuis la rive droite du torrent Cosa. Une partie du dépôt du remplissage de l'abri II se distingue, encore en place.

Figure 1 - Position of the Grotte Verdi of Pradis in the Carnic Pre-Alps. Below left, map of the three shelters with profile of shelters I and II. Right, a picture of the three shelters taken from the right bank of the Cosa stream gorge. A portion of the deposit of shelter II are still in place.

\section{Les grottes Verdi et les fréquentations épigravettiennes du haut plateau de Pradis}

Depuis longtemps reconnu pour son patrimoine spéléologique, le haut plateau de Pradis, ouvert en terrasse vers la plaine du Frioul, a fait l'objet de nombreuses prospections spéléologiques qui ont permis la découverte de restes d'ours des cavernes et d'autres mammifères. Dans ce cadre, les grottes Verdi se présentent comme trois abris qui s'ouvrent en succession sur le côté gauche de la gorge creusée par le torrent Cosa (fig. 1). Ils renfermaient de puissants dépôts de remplissage mais, malheureusement, ces dépôts ont été presque entièrement détruits en 1962 par le curé T. Cattaruzza afin de créer un lieu touristique, de culte et de repos. La mise au jour de restes fauniques et d'artefacts paléolithiques, dans les déblais issus de la vidange des cavités, a conduit la Soprintendenza alle Antichità d'Aquileia, à demander au professeur G. Bartolomei d'effectuer trois brèves campagnes de fouille, en 1970 et 1971, dans les dépôts épargnés par les déblaiements (Bartolomei et al. 1977 ; Corai 1980).

Des trois abris, l'abri I constitue le site majeur en raison de la présence de dépôts pléistocènes d'une épaisseur d'environ deux mètres, mais limités à une surface d'un peu plus de trois mètres carrés au fond de la grotte (fig. 1), contre la voûte de l'abri (fig. 2). La succession stratigraphique s'articule en un ensemble d'unités principalement formées par l'accumulation de graviers cryoclastiques, avec un contenu variable de limons et de niveaux de guano. La géométrie de ces unités est complexifiée par des lentilles dérivées de l'accumulation localisée de graviers cryoclastiques en correspondance avec des niches de la voûte. Les dépôts sont incohérents et partiellement concrétionnés, de manière discontinue. À la base, se trouve une épaisse couche de graviers avec une abondante matrice limono-argileuse. Les niveaux 13 à 7, décapés par niveaux artificiels, contenaient presque exclusivement des restes d'ours des cavernes et quelques rares artefacts lithiques moustériens. Au-dessus, un niveau de guano (6) est couvert de graviers pauvres en limon (5) puis de graviers plus limoneux (couches 4 à 2). Au sommet, les couches 2 et 1 (cette dernière étant subdivisée en $1 a$ et $1 b$ ) abondent en restes de marmotte. Une datation radiométrique (Azzi et Gulisano 1979) sur des charbons de la couche 1a $(12895 \pm 294$ cal BP) place les deux clavicules incisées de marmotte dans le Dryas récent, à la fin de l'Epigravettien récent. Les micromammifères montrent le passage d'une prairie continentale froide à Microtus sp. (arvalis + agrestis) et Microtus nivalis avec Sicista, à une prairie alpine à Microtus nivalis dominant avec Sorex à partir du niveau 2 (Bartolomei 2005).

Les clavicules ont été découvertes en association avec une industrie lithique, deux poinçons en os et des charbons dispersés, non associés à une structure de combustion visible. Le premier poinçon, qui présente une fragmentation d'origine post-dépositionnelle, a été obtenu à partir d'un télémétacarpien d'élan, dont la pointe a été façonnée par raclage. Le second provient de l'ulna gauche d'un canidé (probablement du loup) modifié par raclage 


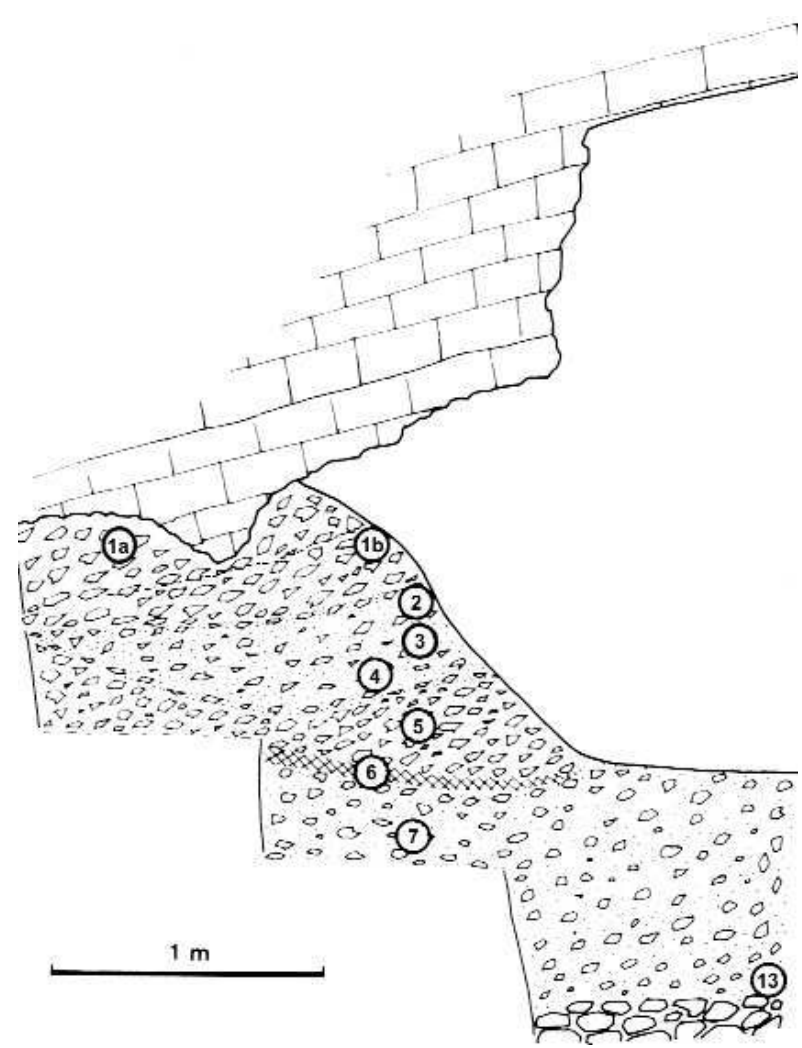

Figure 2 - Section stratigraphique des dépôts de l'abri I.

Figure 2 - Shelter I, stratigraphic section.

afin de former une pointe conique. Son extrémité présente une fracture en languette causée par une flexion excessive durant l'utilisation. L'absence des déchets de façonnage suggère qu'au moins un des deux poinçons a été introduit dans l'abri sous forme d'outil fini (Gurioli 2004). Les niveaux 1 et 2 ont livré une quarantaine de restes de débitage lamellaire, huit outils du fond commun (grattoirs frontaux, burins, troncatures), plusieurs lamelles et six armatures représentées par une lamelle à dos et troncature et des pièces à dos fragmentaires. Deux nucléus à lamelles très réduits sont présents. Le silex est allochtone ; il provient probablement des bassins des Préalpes Vénètes (Corai 1980).

L'association faunistique est représentée presque exclusivement par Marmota marmota, à laquelle s'ajoutent quelques restes attribuables à Ibex ainsi qu'à des cervidés de grande taille. Longtemps interprété comme une accumulation d'origine naturelle (Bartolomei et al. 1977), cet ensemble faunique résulte en réalité d'un apport anthropique primaire, d'après une première analyse des collections de comparaison conservées au Dipartimento di Biologia ed Evoluzione à Ferrare. Cela est confirmé par les nombreuses traces observées sur les mandibules, les clavicules et d'autres éléments anatomiques, qui mettent en évidence des gestes variés et délibérés sur les marmottes adultes et sur quelques restes d'ongulés. Grâce à l'excellent état de conservation des surfaces osseuses, il est possible d'analyser les stries de boucherie afin de reconstituer les modalités d'exploitation des proies et les insérer dans un contexte économique plus large qui rassemble les fréquentations épigravettiennes de ce haut plateau dans la région préalpine orientale. Pour cette raison, nous envisageons un réexamen exhaustif et détaillé de la série, à travers notamment la détermination taxinomique, le décompte total des restes (estimé pour l'instant à 4000 environ, provenant d'à peu près 230 individus d'après le nombre des hémimandibules droites) et surtout l'analyse taphonomique des surfaces osseuses.

\section{Les clavicules de marmotte avec modifications d'origine anthropique}

\section{Objectifs et méthode}

L'étude morpho-technologique des deux clavicules a pour objectif la reconstitution de la séquence de formation des entailles et de contrôler la présence d'éventuelles traces anthropiques mineures, afin de discuter l'hypothèse de modifications produites durant les processus de boucherie.

La détermination de l'âge des individus d'où proviennent les clavicules est basée sur le degré de fusion des épiphyses et la comparaison des dimensions, notamment de la longueur, avec des exemplaires actuels issus de la collection de comparaison.

Les deux clavicules ont été observées sous un stéréomicroscope (Leica MZ8, 10-64x). L'analyse au microscope électronique à balayage (Cambridge Stereoscan S-360) a été effectuée uniquement sur le premier spécimen (fig. 5), afin d'éviter d'éventuels endommagements. Une réplique de la surface portant les stries a été réalisée à partir d'une empreinte négative en élastomère silicone (Provil, Bayer, Leverkusen) puis d'une copie positive sur une résine époxydique (Araldite LY 554, Cyba-Geigy, Bâle). Préalablement à l'observation au MEB, la réplique a subi une métallisation à l'or-palladium (Métalliseur Automatic Hr Sputter Coater-Assing).

\section{Résultats}

À titre de comparaison avec les deux éléments analysés, 42 clavicules issues du niveau 1 ont été observées au préalable. Quinze exemplaires de ce lot montrent des traces de boucherie. Cela correspond à un nombre minimal de 28 individus $(\mathrm{NMI}=28$, calculé à partir du côté droit $=26$, et de l'âge $=2$ ). Onze spécimens $(9+2)$ conservent des traces de modifications anthropiques sur leurs surfaces. Les pièces sont complètes ; leur état de conservation est en règle générale excellent, malgré les quelques traces laissées par des racines et de petits carnivores. Les éléments de modification anthropique correspondent à des stries de boucherie, nombreuses et groupées, perpendiculaires ou obliques à l'axe longitudinal de l'élément anatomique. Elles sont présentes à proximité des deux zones articulaires, la zone proche du sternum présentant des stries profondes, obliques ou perpendiculaires (fig. 3 et 4). Dans tous les cas, les stigmates sont liés au passage répété de l'outil lithique au même endroit ; les stries s'entrecroisent ou se superposent rarement. 

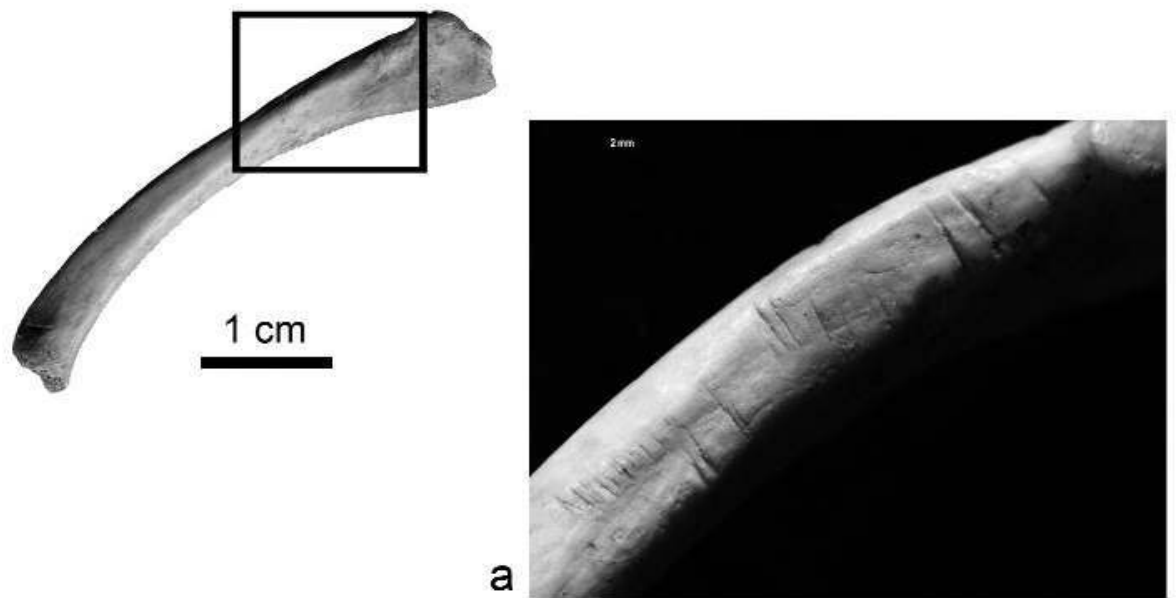

Figure 3 - Deux exemples de clavicules droites de marmotte (Marmota marmota) issues de l'ensemble faunique du niveau 1a : a) pièce 562 (10x), plus de 20 marques de découpe parallèles, très légères, à l'angle entre la face antérieure et la face dorsale, dans la partie acromiale ; b) pièce 564 (12x), au moins 3 marques de découpe sur l'épiphyse sternale, sur la face antérieure.

Figure 3 - Two examples of right marmot (Marmota marmota) clavicles selected from the faunal assemblage of layer 1a : a) bone 562 (10x) showing more than 20 parallel and shallow cut-marks produced during defleshing, at the junction between the frontal and the dorsal faces, on the acromion ; b) bone 564 (12x) showing at least 3 defleshing cut-marks on the epiphysis near the sternum, on the frontal face.

\section{b}

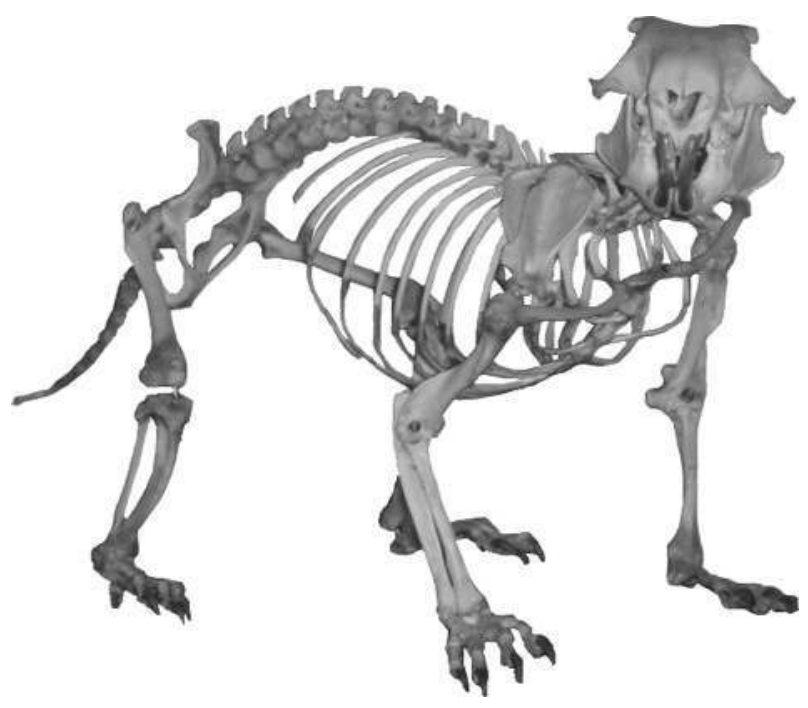

Figure 4 - Squelette de marmotte sur lequel a été mise en évidence la position des deux clavicules.

Figure 4 - Marmot skull with the clavicles marked out.
Les deux clavicules incisées appartiennent à deux individus âgés de deux à trois ans $(43 \times 6 \times 3 \mathrm{~mm}-1$; $43 \times 6 \times 4 \mathrm{~mm}-2)$ dont le sexe reste indéterminable. Elles sont latéralisées à droite et présentent quelques incisions le long du fût et sur l'extrémité sternale. Les surfaces osseuses ne présentent aucune trace attribuable à la prédation naturelle ou aux altérations thermiques.

La première (fig. 5.1) présente cinq entailles initiales, dont quatre ont été creusées à nouveau suivant un axe décalé d'environ $30^{\circ}$ par rapport au précédent (fig. 5.3, 5.4). Trois d'entre elles se trouvent sur le fût, sur le côté antéro-dorsal de l'élément, presque équidistantes les unes des autres. La quatrième, plus éloignée, se situe sur l'extrémité sternale, sur la même face. Les cinq entailles initiales sont profondes et chacune résulte de plusieurs mouvements de sciage parallèles, légèrement décalés afin de couvrir une plus grande superficie. Les entailles suivantes sont formées d'un sillon unique (ou au maximum deux) plus profond que les précédents. II n'est pas possible d'individualiser avec certitude la séquence de production des entailles : les cinq premières ont probablement été incisées dans une même phase puis, dans un second temps, les quatre autres qui s'y superposent. 

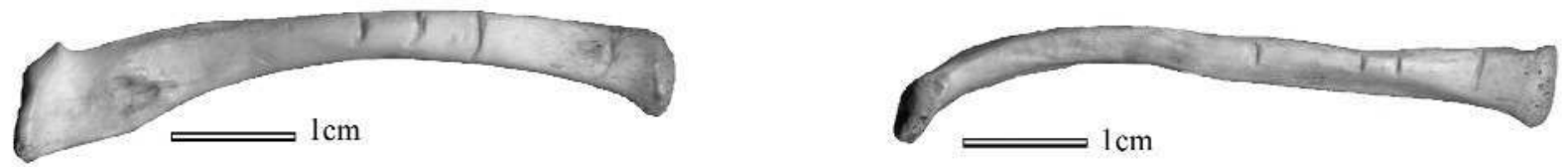

1

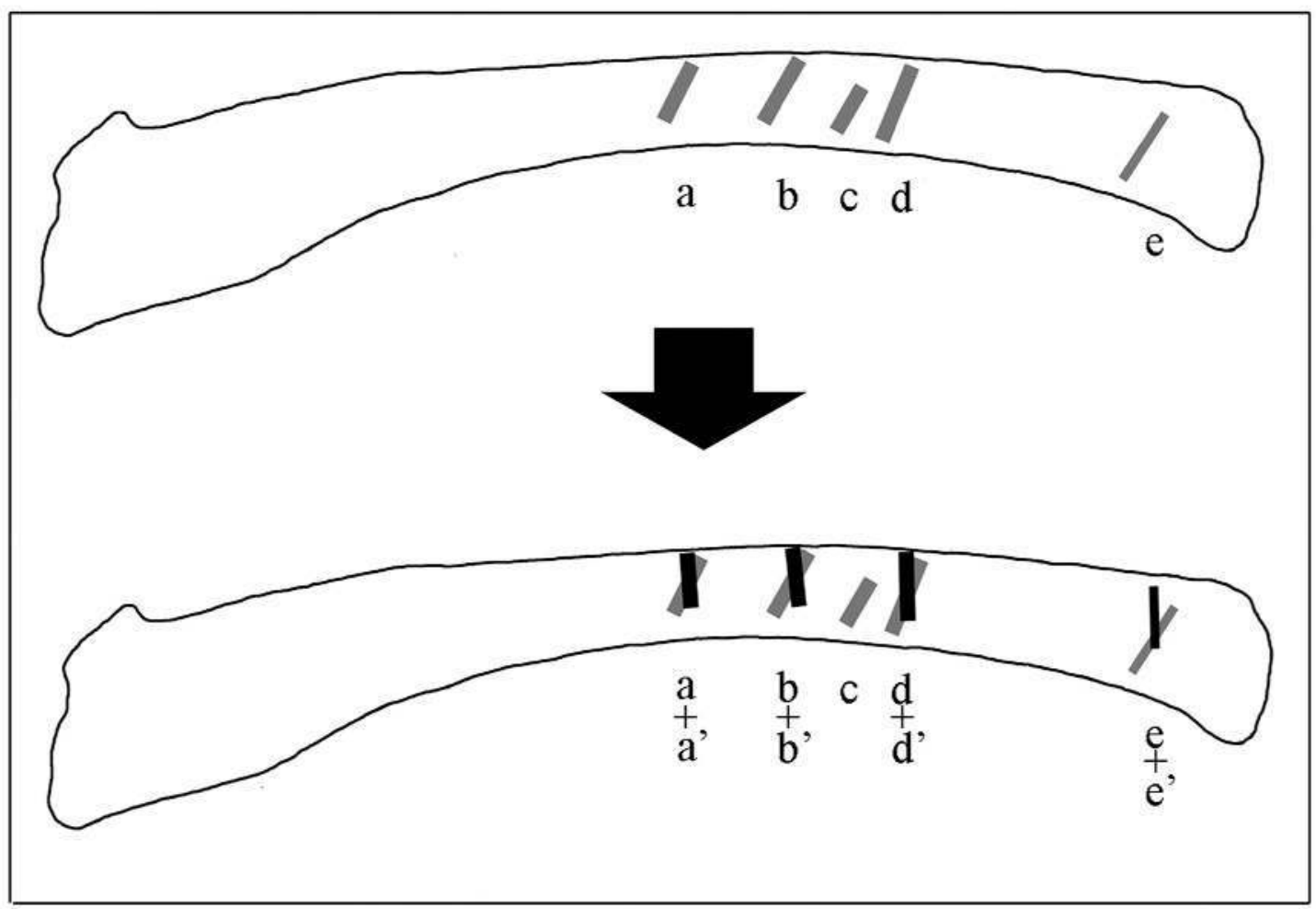

3

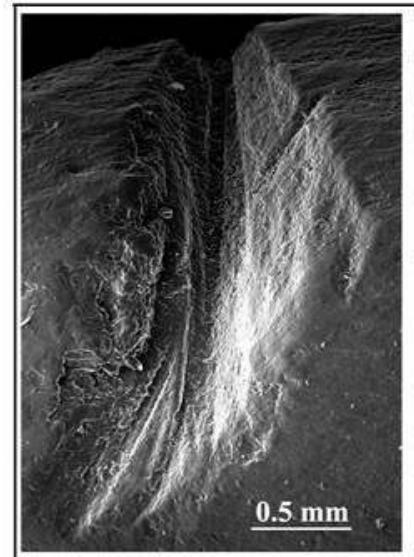

$\mathrm{a}+\mathrm{a}$

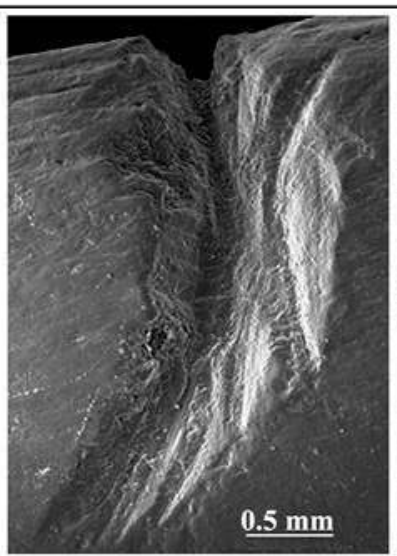

$\mathrm{b}+\mathrm{b}^{\prime}$

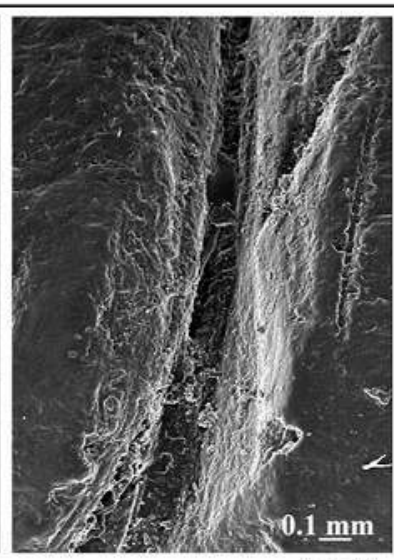

$d+d^{\prime}$

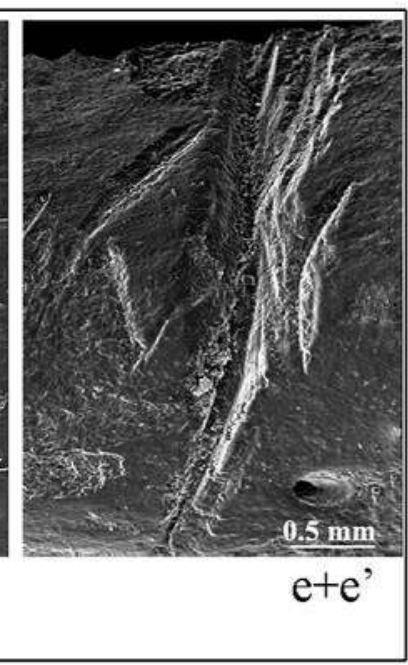

Figure 5-5.1: Clavicule droite de marmotte avec un groupe de quatre entailles concentrées sur le fût et une cinquième sur l'extrémité acromiale. 5.2 : Clavicule droite de marmotte avec deux entailles rapprochées au centre et deux autres latérales, plus distantes. 5.3 :

Schéma opératoire de la production des entailles relevées sur la première clavicule (en gris : entailles initiales, en noir : entailles postérieures). 5.4 : Détail au microscope électronique à balayage de chaque entaille. À noter, la superposition des stries produites par les différentes directions d'incision.

Figure 5 - 5.1 : Marmot right clavicle with four cuts grouped on the shaft and a fifth one visible on the acromial extremity. $5.2:$ Marmot right clavicle with two cuts in the middle and two on the sides, positioned less closer than the formers. $5.3:$ Operative scheme showing the steps in the making of the scores on the first clavicle (grey: initial scores; black: successive scores). 5.4 : SEM view of each score. Note the overposition of the cuts made with different directions. 
La seule entaille à ne pas avoir été creusée une seconde fois se positionne de façon asymétrique entre les trois marques équidistantes. Les entailles qui se situent sur l'extrémité sternale (aussi bien l'entaille initiale que la suivante) ont un sillon plus étroit que les précédentes, en lien probablement avec la nature rugueuse de cette partie de l'os, qui accroche au passage l'outil lithique l'empêchant de glisser correctement.

La seconde clavicule (fig. 5.2) présente quatre entailles, toutes présentes sur la face antérieure. Les deux centrales sont resserrées, alors que celles relevées sur l'extrémité sternale (fig. 5.2, trace de droite) et sur le corps de l'os (fig. 5.2, trace la plus à gauche) sont plus éloignées. Les quatre entailles forment une structure symétrique. L'analyse au stéréomicroscope permet de remarquer sur cet élément quelques concrétions à l'intérieur des entailles, masquant parfois les stries secondaires dans le sillon. Sur la superficie de l'os, des cupules de corrosion sont également présentes ; elles augmentent de diamètre à proximité des extrémités.

\section{Discussion}

Les incisions relevées sur les deux clavicules ont donc été produites intentionnellement avec un mouvement de sciage, au moyen d'un outil lithique. II est exclu qu'il s'agisse de traces liées aux actions effectuées pour l'obtention de la fourrure ou à l'exploitation alimentaire. En effet, les traces de boucherie observées sur les clavicules du même ensemble et sur celles de marmottes issues de plusieurs sites [grotte Colomb (Méaudre, Isère), grotte de La Chênelaz (Hostias, Ain) dans le massif du Vercors (Tomè et Chaix 2003), mais aussi la grotte du Clusantin sur le même haut plateau de Pradis (Romandini et al. sous presse)], sont généralement caractérisées par un, ou plusieurs gestes, témoignant d'un unique passage du tranchant du silex, qui ne se répète jamais au même endroit. Les stries, souvent obliques par rapport à l'axe majeur de l'élément anatomique, se croisent rarement et sont toujours caractérisées par un sillon en « V » peu ou moyennement profond.

De plus, il faut souligner que les deux spécimens présentent une série initiale d'incisions comparables, alors qu'un seul exemplaire porte un second groupe. Pour au moins l'une d'entre elles (clavicule 1 - fig. 5.1), nous pouvons exclure qu'il s'agisse de stries créées sur l'élément anatomique en connexion. Cela aurait empêché une action de sciage efficace sur la face antéro-ventrale. En outre, nous soulignons que sur la première clavicule, la seule entaille à ne pas avoir été creusée une seconde fois se positionne de façon asymétrique entre les trois marques équidistantes : une raison esthétique est probablement à la base de ce choix.

Le cas de la seconde clavicule est différent (clavicule 2 fig. 5.2) : la position des sillons sur le côté antérieur et leur orientation transversale par rapport à l'axe longitudinal de l'élément squelettique ne peuvent pas exclure une première formation des traces durant les phases de boucherie. L'âge des individus correspond parfaitement aux objectifs de prédation anthropique, focalisée sur la teneur maximale en viande et en graisse des proies. La différence d'état de conservation observée entre les deux spécimens, vu la légère corrosion et les concrétions carbonatées sur la clavicule 2, reste vraisemblable au sein d'une même couche, surtout en grotte.

Le complexe des grottes Verdi de Pradis n'est pas le seul site lié à la marmotte sur le haut plateau. L'intérêt pour ce rongeur de la part des populations épigravettiennes est bien documenté dans la grotte du Clusantin, sur le côté occidental d'une doline située à quelques centaines de mètres des grottes Verdi. La position de cette petite cavité, le profil techno-économique et fonctionnel de l'ensemble lithique et les études archéozoologiques démontrent que l'accumulation des restes squelettiques de marmotte, qui dominent presque totalement l'association faunistique, résulte de l'apport saisonnier de carcasses d'âge varié et de leur exploitation à des fins alimentaires ou utilitaires (Peresani et al. 2008 ; Peresani et al. sous presse). Les traces présentes sur les ossements renvoient aux différentes étapes de traitement de la carcasse, axées sur le démembrement et sur le détachement des masses carnées, mais aucune évidence n'atteste d'un quelconque intérêt non utilitaire.

\section{Contextualisation culturelle et conclusion}

L'ensemble des traces relevées sur les deux clavicules de marmotte a conduit à considérer que ces éléments anatomiques ont servi de support d'objet à caractère non utilitaire. Leur unicité dans l'Epigravettien récent et les autres cultures contemporaines du Tardiglaciaire en font un protagoniste de premier ordre, dans la mesure où ces pièces n'ont pas d'équivalent. II existe d'autres éléments anatomiques de marmotte qui ont été modifiés sans but fonctionnel, comme c'est le cas des incisives sciées mises au jour sur les sites magdaléniens. Toutefois, le peu de soin apporté à la régularisation des surfaces sciées, ainsi que le manque de standardisation dimensionnelle, irait dans le sens d'une utilisation comme amulettes plutôt que comme éléments décoratifs, peut-être en raison de la couleur orange de leur émail (Bullinger et Müller 2005). Les auteurs n'excluent cependant pas que ces dents aient fait partie de la gamme ordinaire de la parure magdalénienne.

En ce qui concerne l'unicité des clavicules de Pradis, dans la mesure où les traces relevées sont relativement peu visibles, il n'est pas exclu que des formes similaires puissent être présentes dans de vieilles collections qui n'ont pas encore été étudiées. En effet, le mobilier funéraire de cette phase finale du Paléolithique supérieur comprend également des restes osseux de petits animaux comme le hérisson, le lapin, le castor et l'écureuil. Ils se situent parfois directement sur le corps du défunt, attestant d'une intentionnalité dans le prélèvement de certaines portions anatomiques précises, telles que les mandibules, les vertèbres caudales et les membres (Cardini 1980 ; d'Errico et Vanhaeren 2000 ; Vanhaeren et d'Errico 2001). Les exemples les plus importants proviennent des nécropoles de la grotte d'Arene Candide (Cardini 1980) et de l'aven des Iboussières (d'Errico et Vanhaeren 2000) ; ce dernier ayant livré cinq mandibules 
de hérisson et deux de lapin, décorées d'entailles régulièrement espacées à la base de l'élément anatomique.

Les conditions dans lesquelles ont été mises au jour les deux clavicules permettent de diriger les interprétations vers les systèmes d'annotations, les amulettes et les objets ornementaux d'usage quotidien, qu'il s'agisse d'objets finis ou d'ébauches d'extraction. Leur association nous paraît peu probable avec un contexte funéraire dont l'abri ne garde aucune trace malgré la faible surface de la zone fouillée.

\section{Remerciements}

Nous remercions la Soprintendenza per i Beni Archeologici du Frioul - Vénétie Julienne pour nous avoir autorisé à étudier le matériel, la conservatrice archéologue Anna Nicoletta Rigoni, du Musée des Sciences de Pordenone, pour sa disponibilité lors du prélèvement des pièces, B. Sala et U. Thun Hohenstein du Département de Biologie et Evolution de I'Università degli Studi di Ferrara (Italie), pour nous avoir permis d'utiliser la collection ostéologique de comparaison et pour l'aide fournie lors de la préparation des répliques en résine. Cette recherche a été réalisée avec le concours de la Società Naturalisti «S. Zenari ». Texte traduit de l'italien par Camille Jéquier et révisé par Jean-Pierre Chadelle. Deux rapporteurs anonymes ont proposé des suggestions fondamentales pour améliorer ce manuscript.

\section{Références bibliographiques}

AZZI C.M. et GULISANO F. 1979 - Florence Radiocarbon Dates IV. Radiocarbon XXI, p. 353-357.

BARTOLOMEI G. 2005 - Riparo Cogola (1075 m), Carbonare di Folgaria, Trento: indicazioni paleoecologiche dai reperti di micromammiferi di una località montana e considerazioni sugli ambienti delle fasce altimetriche. Preistoria Alpina 41, p. 51-63.

BARTOLOMEI G., BROGLIO A. et PALMA DI CESNOLA A. 1977 - Chronostratigraphie et écologie de l'Epigravettien en Italie. In : La fin des temps glaciaires en Europe Chronostratigraphie et écologie des cultures du Paléolithique final. C.N.R.S. 271, p. 297-324.

BOLLINGER J. et MÜLLER W. 2005 - Nouvelles découvertes d'incisives de marmotte sciées dans des sites magdaléniens de l'arc jurassien. In : Industries osseuses et parures du Solutréen au Magdalénien en Europe. Mémoires de la Société Préhistorique Française, XXXIX, p. 347-351.

BROCHIER J.-É. et BROCHIER J.-L. 1973 - L'art mobilier de deux nouveaux gisements magdaléniens à Saint-Nazare-enRoyans (Drôme). Études Préhistoriques 4, p. 1-12.

CARDINI L. 1980 - La necropoli mesolitica delle Arene Candide. Memorie dell'Istituto Italiano di Paleontologia Umana 3, p. 9-31.
CORAI P. 1980 - Le più antiche culture preistoriche della "Ladinia » (Paleolitico e Mesolitico). Ladinia - Sföi Culturâl Dai Ladins Dles Dolomites 4, p. 183-218.

D'ERRICO F. et VANHAEREN M. 2000 - Mes morts et les morts des mes voisins. Le mobilier funéraire de l'Aven des lboussières et l'identification de marqueurs culturels à l'Épipaléolithique. In : A. Richard, C. Cupillard, H. Richard et $A$. Thévenin (eds), Les derniers chasseurs-cueilleurs d'Europe occidentale. Annales Littéraires 699, Environnement, Sociétés et Archéologie 1, p. 325-342.

D'ERRICO F., ZILHAO J., BAFFIER D., JULIEN M. et PELEGRIN J. 1998 - Neanderthal acculturation in Western Europe? A critical review of the evidence and its interpretation. Current Anthropology 39, p. 1-44.

DESBROSSE R. 1972 - Les dents incisées du Paléolithique. L'Anthropologie 76/1-2, p. 135-140.

GRIGGO C., MONIN G., FOURNIER J. et OBERLIN Ch. sous presse - Activités de chasses saisonnières à la marmotte au Tardiglaciaire dans le Vercors : implications environnementales, chrono-culturelles et économiques. In : F. Bon, S. Costamagno et N. Valdeyron (eds.), Haltes de chasse en Préhistoire: quelles réalités archéologiques? Université Toulouse Le Mirail.

GURIOLI F. 2004 - I manufatti in osso provenienti dai livelli dell'Epigravettiano recente delle Grotte Verdi di Pradis (Prealpi friulane, PN). Bollettino Società Naturalisti "Silvia Zenari » 28, p. 39-48.

LEESCH D., CATTIN M.-I. et MÜLLER W. 2004 - Témoins d'implantation magdaléniennes et aziliennes sur la rive nord du lac de Neuchâtel. Archéologie neuchâteloise 31.

MONIN G., GRIGGO C. et TOMÉ C. 2006 - Stratégies d'exploitation d'un écosystème alpin au Tardiglaciaire. Les chasseurs de marmottes du Vercors. In : Miras Y. et F. Surmely (eds), Actes de la table ronde de Pierrefort. Presses Universitaires de Franche-Comté, Besançon, p. 29-50.

PATOU-MATHIS M. 1987 - Les marmottes: animaux intrusifs ou gibiers des préhistoriques du Paléolithique. Archeozoologia 1, p. 93-107.

PATOU-MATHIS M., DESBROSSE R. et MARGERAND I. 1991 - Quelques sites préhistoriques à marmottes du Tardiglaciaire dans les Alpes du Nord et le Jura méridional. In : Actes du $116^{\circ}$ Congrès National des Sociétés Savantes. Archéologie et Environnement des Milieux Aquatiques. Comité des Travaux Historiques et Scientifiques, p. 367-392.

PERESANI M., DE CURTIS O., DUCHES R., GURIOLI F., ROMANDINI M. et SALA B. 2008 - Grotta del Clusantin, un sito inusuale nel sistema insediativo epigravettiano delle Alpi italiane. In : M. Mussi (ed), II Tardiglaciale in Italia. Lavori in corso. British Archaeological Reports, International Series 1859 , p. 67-79.

PERESANI M., DUCHES R., MIOLO R., ROMANDINI M. et ZIGGIOTTI S. sous presse - Un exemple de variabilité dans 
l'exploitation des ressources alimentaires épigravettiennes dans les Alpes Orientales italiennes. In : F. Bon, S. Costamagno et $N$. Valdeyron (eds.), Haltes de chasse en Préhistoire: quelles réalités archéologiques? Université Toulouse Le Mirail.

POPLIN F. 1983 - Les dents travaillées de renne et autres animaux de Petersfels. In : G. Albrecht, $H$. Berke et F. Poplin (eds.), Recherches scientifiques sur les inventaires magdaléniens du Petersfels, fouilles 1974-9176. Archaeologica Venatoria, p. 133-153.

ROMANDINI M., PERESANI M., GURIOLI F. et SALA B., sous presse - Marmota marmota, the most predated species at Grotta del Clusantin. Insights from an unusual case-study in the Italian Alps. In: L. Kindler et S. Gaudzinski-Windheuser (eds.), Hominin subsistence in the Old World during the Pleistocene and early Holocene. Quaternary International. DOI: 10.1016/j.quaint.2011.03.016.
TILLET Th. 2001 - Les Alpes et le Jura. Quaternaire et Préhistoire ancienne. GB: Grenoble.

TOME C. et CHAIX L. 2003 - La chasse et l'exploitation des marmottes dans les Alpes occidentales et le Jura du sud de la fin du Pléistocène à l'Holocène. In : $R$. Ramousse, D. Allainé et M. Le Berre (eds.), Stratégies adaptatives et diversité chez les marmottes. International network on Marmots, p. 77-84.

VANHAEREN M. et D'ERRICO F. 2001 - La parure de l'enfant de la Madeleine (fouilles Peyrony). Un nouveau regard sur l'enfance au Paléolithique supérieur. PALEO 13, p. 201-237. 